\title{
Classification of gas reduction valve silencers at TPP gas distribution points
}

\author{
Vitaliy Skvortsov *, Vladimir B. Tupov \\ National Research University "Moscow Power Engineering Institute”, Moscow, Russia
}

\begin{abstract}
Gas distribution points (GDP) at TPPs are sources of intense noise. Results of noise experimental measurements originating from TPP GDP demonstrate that the sound level at a distance of $10 \mathrm{~m}$ could reach 110 $\mathrm{dBA}$. Gas reduction valves are the source of noise in GDP. N oise emission characteristic of the gas reduction valves depends on their geometric and design parameters. To reduce the noise propagating in a low-pressure gas pipeline from gas reduction valves, various types of noise silencers are used. This paper presents results of noise experimental measurements in the vicinity of the GDP buildings at various energy enterprises. Overview and classification of silencers by various manufacturers used at TPP GDPs are provided.
\end{abstract}

\section{Introduction}

Gas reduction valves installed in GDPs are a source of strong noise, which reaches $130 \mathrm{dBA}$ directly near the gas reduction valves and $110 \mathrm{dBA}$ in the vicinity of the GDP building [1-2]. These values exceed sanitary standards [3] for working areas by 30 and $45 \mathrm{dBA}$, respectively. Various methods are used to reduce noise from gas reduction valves, including special devices to reduce noise of the gas reduction valves.

Due to specifics of the GDP operation, these devices are used to solve two major problems:

- reducing vibration load on gas pipelines installed behind the gas reduction valves;

- reducing the level of sound pressure generated during natural gas reduction in the gas reduction valves.

Let us consider solution to the second problem.

\section{Gas reduction valve noise level dependence on TPP capacity}

An increase in the power of the station N, MW, leads to the increase in the B consumption, $\mathrm{kg} / \mathrm{s}$, of gas consumed by a TPP, which could be calculated as:

$$
B=\frac{N}{Q * \eta} \text {, }
$$

where: $\mathrm{Q}$ is the gas net calorific value, $\mathrm{MJ} / \mathrm{kg} ; \eta$ is the power plant efficiency.

* Corresponding author: skvor.vitalya@yandex.ru 
Acoustic power of the $W a$ gas flow, $\mathrm{W}$, passing through the gas reduction valve could be described as an equation, if $\mathrm{M}=0.5 \ldots 1$ [4]:

$$
W_{a}=3 \times 10^{-5} \frac{\rho_{c}^{2} \times \vartheta^{8} \times D^{2}}{\rho_{0} \times a_{3 \mathrm{~B}}^{5}},
$$

where: $\rho_{\mathrm{c}}, \rho_{0}$ are the flow and medium density, $\mathrm{kg} / \mathrm{m}^{3}$;

$v$, as are the speed of medium and sound, respectively, $\mathrm{m} / \mathrm{s}$;

$\mathrm{D}$ is the diameter of flow passing through the gas reduction valve, $\mathrm{mm}$.

As the gas flow rate grows, the TPP gas reduction valve sound power level also increases.

Table 1 presents data characterizing dependence of the sound power level of most widely used valves on the TPP electric power capacity.

Table 1. Sound power levels of GDP gas reduction valves

\begin{tabular}{|c|l|}
\hline TPP capacity, MW & Sound power level, dBA \\
\hline 100 & 113.9 \\
\hline 150 & 115.6 \\
\hline 200 & 116.9 \\
\hline 500 & 120.9 \\
\hline 1,000 & 123.9 \\
\hline 1,500 & 125.6 \\
\hline 2,000 & 126.9 \\
\hline 2,500 & 127.9 \\
\hline 3,000 & 128.7 \\
\hline
\end{tabular}

It could be seen that sound power levels of the GDP gas reduction valves are varying from almost 114 to $129 \mathrm{dBA}$.

It should be noted that sound power level significantly depends on the valve type and its design parameters. In this case, the values may differ significantly from those provided in Table 1.

\section{Reducing the sound level generated during natural gas reduction}

Depending on the operation principle, such devices are called silencers, pressure pulsation dampers, flow dividers and throttle chambers.

The given equation shows that acoustic power of the gas reduction valve flow depends on gas density in the flow, its diameter, and also on the flow speed.

It is known that the control valve oscillatory power increases in proportion to the square of differential pressure and to the ratio of differential pressure to the static pressure. Thus, when the gas flow passes through a number of obstacles, dynamic loads decrease could be obtained [5].

This is the principle behind operation of the most pressure pulsation dampers, flow dividers and throttle chambers.

Flow dividers and throttle chambers are the most common type of devices used to suppress noise emitted during gas reduction. It should be noted that devices for lowering the gas reduction valve noise are also used to reduce vibration load in the gas pipelines installed behind the gas reduction valves.

Figure 1 shows a principle view of a throttle chamber.

Most manufacturers, both domestic and foreign, offer various versions of these noise suppression devices. For example, Tartarini offers SR and SRII type silencers to be installed 
directly into the pressure reducing valve with a declared acoustic efficiency of up to $20 \mathrm{dBA}$. Example of such a device is shown in Figure 2.

Similar flow dividers and throttle chambers are available from other manufacturers, such as Emerson FisherEWD with WHisperFlo finishing or WhisperTrim I Cage by SteamForm.

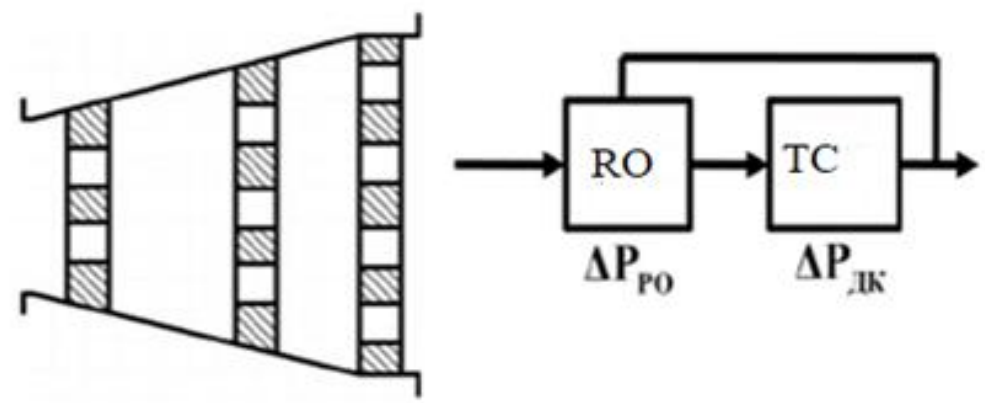

Fig. 1. Throttle chamber
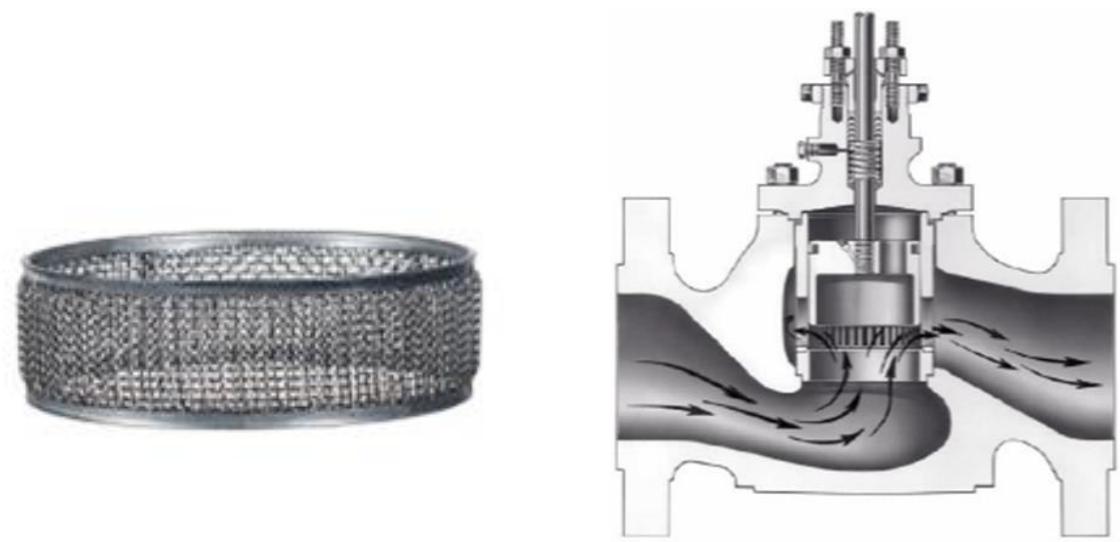

Fig 2. Tartarini SR series silencers

Besides, silencers of SRS and SRSII series with acoustic efficiency of up to $30 \mathrm{dBA}$, which is shown in Figure 3, could be mentioned. 

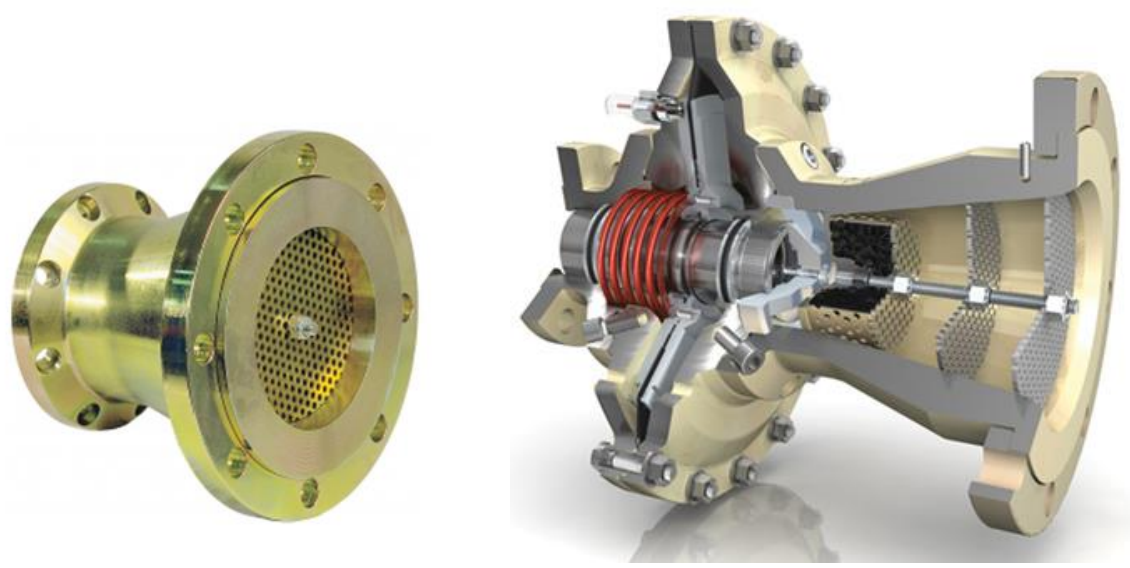

Fig 3. Tartarini SRS and SRSII series silencers

The use of throttle chambers and flow dividers makes it possible to unload the reduction valve regulating body, while the regulator outlet pressure tracking point is displaced behind the throttle chamber. When selecting this type of device, it is necessary to limit their hydraulic resistance in order to ensure that the valve is not losing the ability to regulate the gas flow rate.

Absorption silencers are proposed to use, where the noise level reduction is achieved due to the sound energy dissipation effect on the surface of the sound-absorbing material. For example, an STR Tartarini series silencer with the acoustic efficiency of up to $20 \mathrm{dBA}$ is presented in Figure 4.
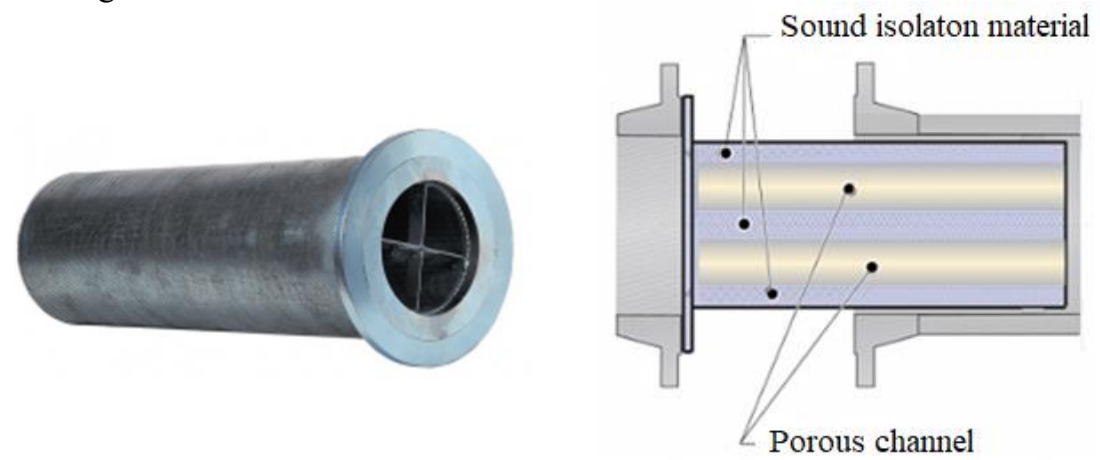

Fig 4. Tartarini STR series silencers

Using the dissipative silencers at GDPs is limited and is not always recommended due to the loss of efficiency because of possible contamination and high hydraulic resistance.

As an example of domestic analogue, the GAZPROMMASH noise suppressor shown in Figure 5 could be introduced, which combines both the properties of throttle chambers and flow dividers, as well the sound energy dissipation effect. 

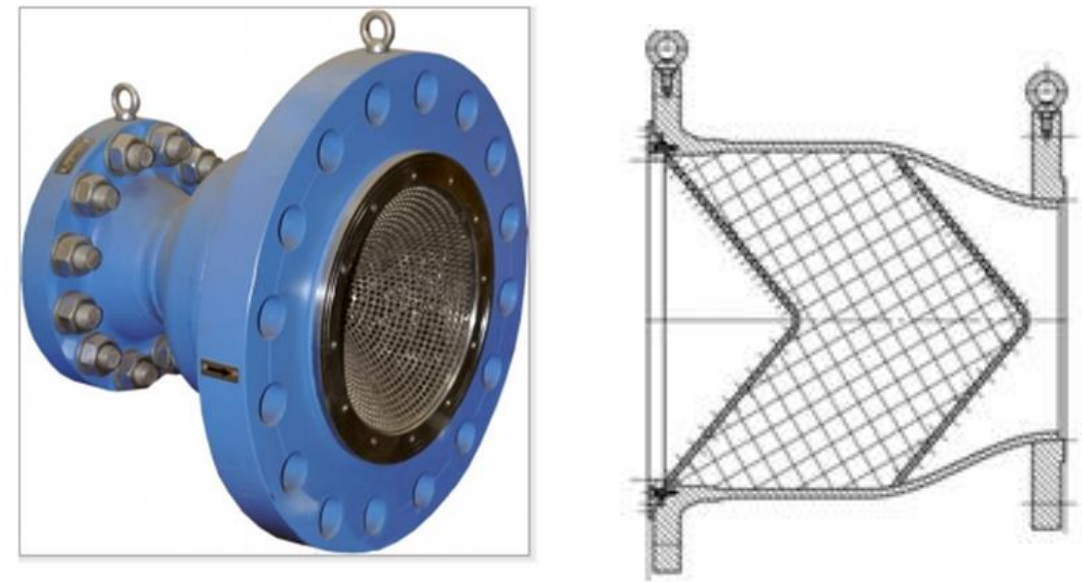

Fig 5. GAZPROMMASH silencer

This silencer with a flow straightener is a metal multi-level prefabricated assembly consisting of steel body parts, perforated diffusers at the inlet and outlet and of a soundabsorbing filler. The silencer acoustic efficiency ranges from 15 to $25 \mathrm{dBA}$. Noise reduction is ensured by both the gas flow fragmentation, altering its direction and by the sound energy dissipation in the sound absorption filler.

Table 2 summarizes information on the acoustic performance of certain valves.

Table 2. Classification of silencers depending on the sound level decrease magnitude, dBA

\begin{tabular}{|c|c|}
\hline Name & Sound decrease level value, dBA \\
\hline GAZPROMMASH NS & $15-25$ \\
\hline $\begin{array}{c}\text { SR and SRII series (Tartarini), } \\
\text { FisherEWD (SteamForm), STR } \\
\text { (Tartarini) silencers }\end{array}$ & 20 \\
\hline $\begin{array}{c}\text { SRS and SRSII (Tartarini) series } \\
\text { silencers }\end{array}$ & 30 \\
\hline
\end{tabular}

Device classification conducted shows possibility of noise reduction in the GDP vicinity by 15-30 dBA. However, such noise reduction is not sufficient for any GDP of the high power TPP, where noise level near the valves reaches 125-130 dBA [1]. To meet the requirements for noise level, according to $\mathrm{CN} 2.2 .4 / 2.1 .8 .562-96$, it should correspond to "noise at workplaces, in residential and public buildings and on the territory of residential buildings" requirements.

Reaching the standard values for noise level at workplaces originating from the GDP gas reduction valves is a complex task, when it is necessary to introduce several measures simultaneously. Such measures could include reducing noise in a valve, soundproofing casing, pipeline soundproofing and installation of noise suppressing barriers in vicinity of a GDP. When using these measures, it should be borne in mind that a sound-absorbing barrier could ensure noise reduction efficiency of up to $20 \mathrm{dBA}$; and sound insulation should meet the conditions of the GDP operation and prevent the gas pipeline corrosion due to condensation of water vapor from the environment [6-9]. 


\section{Conclusion}

1. Noise originating from the gas GDP gas regulation valves appears to be an intense source of noise coming from a TPP. An increase in the TPP load leads to the growing sound level from the gas regulation valves.

2. Achieving standard values on the noise level at workplace from the GDP gas reduction is a complex task, where it is necessary to introduce certain measures simultaneously. Such measures could include reducing the noise in a valve, sound-insulating casing, gas pipeline sound insulation and installing noise suppression barriers in vicinity of a GDP building.

\section{References}

1. V.S. Skvortsov, V.B Tupov, Noise from TPP GDP in the total noise level at the SPZ boundary, in Proceedings of the Fourth A II-R ussian Conference of $Y$ oung Scientists and Specialists "Acoustics of the Environment" (A SO-2019), B M STU, 24 M ay 2019, Moscow, Russia (2019)

2. V.B. Tupov, B.V. Tupov, V.S. Skvortsov, Power Technology and Engineering, 52(4), $448-450$ (2018)

3. SN 2.2.4/2.1.8.562-96. Noise at workplaces, in residential, public buildings premises and in territories of residential construction. - M .: M inistry of Health of Russia (1997)

4. V. I Golovanov, V. N. Slavyaninov, V. K. Fedorov, Noise silencer for gas jets with large flow rate, Electronic Journal "Technical Acoustics”, http://www.ejta.org, 9 (2002)

5. D. Jury Floyd, Fundamentals of aerodynamic noise in control valves. M arshalltown, IA, Fisher Controls International Inc. (1999)

6. V. Tupov, B. Tupov, Solutions to creating a low-noise power facility, in Proceedings of the 24th International Congress on Sound and V ibration, 23-27 J uly 2017, L ondon, UK (2017)

7. S.A. Chuvirova, V. B. Tupov, Possibilities of acoustic barriers for reducing the GDP and gas pipelines noise following it, in Proceedings of the V II A II-R ussian Scientific and Practical Conf. with int. participation, M arch 19-21 2019, St. Petersburg Russia, (2019)

8. V.B Tupov, S.A Semin, B.V. Tupov, A. A. Taratorin, D.A. Rozanov, Power Technology and Engineering 50(6), 649-652 (2017)

9. V. B. Tupov, S. A. Semin, B. V. Tupov, A. A. Taratorin, D. A. Rozanov, New in the Russian electric power industry, 11, 39-48 (2016) 